\title{
BMJ Open Evaluation of the feasibility and impacts of in situ simulation in emergency medicine-a mixed-method study protocol
}

\author{
Jennifer Truchot, ${ }^{1,2,3}$ Valérie Boucher, ${ }^{2,4}$ Éliane Raymond-Dufresne, ${ }^{3}$ \\ Christian Malo, ${ }^{1,3}$ Éric Brassard, ${ }^{3}$ Jean Marcotte, ${ }^{3}$ Guillaume Martel, ${ }^{1}$ \\ Geneviève Côté, ${ }^{1,3}$ Christian Garneau, ${ }^{3}$ Gino Bouchard, ${ }^{3}$ Marcel Emond (1) ${ }^{3,4}$
}

To cite: Truchot J, Boucher V, Raymond-Dufresne É, et al. Evaluation of the feasibility and impacts of in situ simulation in emergency medicine-a mixed-method study protocol. BMJ Open 2021;11:e040360. doi:10.1136/ bmjopen-2020-040360

- Prepublication history and additional materials for this paper is available online. To view these files, please visit the journal online (http://dx.doi. org/10.1136/bmjopen-2020040360).

Received 11 May 2020 Revised 15 February 2021 Accepted 24 February 2021

Check for updates

(C) Author(s) (or their employer(s)) 2021. Re-use permitted under CC BY-NC. No commercial re-use. See rights and permissions. Published by BMJ.

1Département de médecine familiale et de médecine d'urgence, Université Laval Faculté de médecine, Quebec, Québec, Canada

${ }^{2}$ Research center- CHU de Québec, Université Laval, Quebec, Québec, Canada ${ }^{3}$ Emergency Department, CHU de Québec-Université Laval, Quebec, Québec, Canada

${ }^{4}$ VITAM - Centre de recherche en santé durable, Quebec, Québec, Canada

Correspondence to

Dr Marcel Emond;

marcel.emond@fmed.ulaval.ca

\section{ABSTRACT}

Introduction In situ simulation (ISS) consists of performing a simulation in the everyday working environment with the usual team members. The feasibility of ISS in emergency medicine is an important research question, because ISS offers the possibility for repetitive, regular simulation training consistent with specific local needs. However, ISS also raises the issue of safety, since it might negatively impact the care of other patients in the emergency department (ED). Our hypothesis is that ISS in an academic high-volume ED is feasible, safe and associated with benefits for both staff and patients. Methods A mixed-method, including a qualitative method for the assessment of feasibility and acceptability and a quantitative method for the assessment of patients' safety and participants' psychosocial risks, will be used in this study.

Two distinct phases are planned in the ED of the CHU de Québec-Université Laval (Hôpital de l'Enfant-Jésus) between March 2021 and October 2021. Phase 1: an ISS programme will be implemented with selected ED professionals to assess its acceptability and safety and prove the validity of our educational concept. The number of cancelled sessions and the reasons for cancellation will be collected to establish feasibility criteria.

Semistructured interviews will evaluate the acceptability of the intervention. We will compare unannounced and announced ISS. Phase 2: the impact of the ISS programme will be measured with validated questionnaires for the assessment of psychosocial risks, self-confidence and perceived stress among nonselected ED professionals, with comparison between those exposed to ISS and those that were not.

Ethics and dissemination The CHU de QuébecUniversité Laval Research ethics board has approved this protocol (\#2020-5000). Results will be presented to key professionals from our institution to improve patient safety. We also aim to publish our results in peerreviewed journals and will submit abstracts to international conferences to disseminate our findings.

\section{INTRODUCTION}

Simulation is a teaching tool used for the acquisition of technical and nontechnical
Strengths and limitations of this study

- This study is the first to simultaneously assess the acceptability, feasibility and safety of conducting in situ simulation (ISS) in a busy academic emergency department.

- The issue of patient safety during ISS is an important ethical consideration, which is rarely included in the design of simulation research.

- Even though ISS offers the possibility of improving patient safety through training, it can also jeopardise the quality of ongoing care by redirecting human resources from patients to the training process.

- The positive impact of ISS on patient outcomes was highlighted in a systematic review, however, without mention of patients' safety.

- As it often is the case in simulation studies, the scope of our results might be restrained by methodological limitations such as the absence of randomisation and the inability to blind participants to the outcome.

skills. ${ }^{1}$ Numerous studies have shown that simulation is associated with a significant beneficial effect for every health professional. $^{23}$ This is also the case in emergency medicine (EM), which is a complex interprofessional specialty that requires a broad range of clinical knowledge as well as the mastery of multiple technical (ie, intubation, chest tube insertion, lumbar puncture) and nontechnical clinical skills (ie, communication, task distribution, leadership and followership). Simulation enables new skill acquisition as well as continuing clinical education and training, which are necessary to manage both everyday situations and rare clinical cases. A wide variety of simulation aids exist, ranging from simple task manikins to virtual reality or hybrid simulation using live actors and manikins to increase realism. ${ }^{4}$ The choice of the right tool must be based on a proper balance 
between learning objectives and the required level of realism. Realism, also called fidelity in simulation, ${ }^{5}$ greatly impacts the quality of learning and especially the transfer of these skills to the real clinical world. ${ }^{6}$ However, educators must carefully examine the stress generated by the simulation exercise. Stress can limit skill acquisition if the exercise is too complex for the participant's qualification and previous experience. ${ }^{78}$

Simulation training can take place in a dedicated centre that is sometimes located off site, which consequently limits the training's wide implementation. Simulation centres necessitate human resources and structural expenditures, resulting in significant costs. The conditions they simulate are also quite different from those of the emergency department (ED) environment, thus decreasing the realism of the training.

It has also become increasingly difficult to get participants to engage in regular simulation training, especially when it is set to take place far from their usual workplace and outside usual working hours. ${ }^{9-11}$ This is a problem for knowledge and skill retention given that effective learning seems to require repetition in training. ${ }^{12}$ For example, the use of simulation is now recommended to teach and train basic and advanced life support. ${ }^{13} \mathrm{~A}$ recent study found that the optimal training frequency for adequate retention of cardiopulmonary resuscitation was once a month. ${ }^{14}$ However, training all ED staff every month is impossible for most institutions.

In situ simulation (ISS), a type of simulation integrated into the targeted clinical environment, is a practical solution to these issues. The rationale for using it is based on the importance of environmental fidelity and its potential impact on learning. ${ }^{15}$ Studies comparing simulation in a dedicated centre to ISS are scarce. This could be due to the complex methodology required to implement them. ${ }^{16}$ Nevertheless, a 2015 randomised study highlighted that participants' perception of realism was considerably higher in the in situ group. ${ }^{16} 17$

ISS also offers the interesting possibility of identifying conditions-known as latent safety threats (LST) - that can lead to errors in the usual working environment. ${ }^{18}$ LSTs are 'system-based threats to patient safety that can materialise at any time and are previously unrecognised by healthcare professionals and/or hospital administration'. ${ }^{19}$ One of the benefits of ISS is that since the simulations occur in a real-life environment, they enable the identification of LSTs such as equipment malfunctions or suboptimal team organisation and responsibility awareness. This leads to a better understanding of potential errors and, therefore, to the possibility of reducing their incidence. ${ }^{18}$ Numerous studies illustrate the positive impact, including improved patient outcomes, of ISS on the clinical practice of healthcare professionals from various specialties. ${ }^{20-23}$ However, the complex environment of an ED can be challenging for those conducting ISS training. The difficult work conditions (overcrowding, task interruptions, understaffing) in EM can be a major practical limit to the implementation of ISS training. ${ }^{24}$ The literature exploring different types of ISS is still scant. ${ }^{25}$ Different modalities have been compared through qualitative analysis and mostly using self-assessment tools. ${ }^{25}$ For example, some authors compared unannounced ISS (outside of the scheduled work period) to announced ISS (during the scheduled work period) and found no difference in terms of preference or stress. These findings should be confirmed with objective and validated assessment tools combined with the exploration of more practical considerations, such as the safety of ISS itself. The ISS process can be used to enhance patient safety. ${ }^{26}$ Simulation is an effective strategy for training many skills-including, as in this case, handover communication skills. Our work aims to confirm the innocuousness of ISS training. ISS is often used as a tool to improve the quality and safety of patient care, but it might also cause harm by redirecting resources and attention from patient care to the training process.

Therefore, our study will explore different modalities of exposure to ISS in the ED: no ISS (control group), announced ISS (outside the work shift) and unannounced ISS (unexpected simulation during the work shift).

\section{Objectives}

Primary objective

Phase 1: to assess and compare the feasibility of two types of ISS in the ED: announced and unannounced.

Phase 2: to assess whether ISS improves participants' psychosocial impact (stress reduction, satisfaction and self confidence improvement) when compared with no exposure to ISS (control group).

Secondary objectives

1. To assess and compare the safety of two types of ISS in the ED: announced and unannounced.

2. To compare the number of LST identified during unannounced ISS and announced ISS. ${ }^{13}$

\section{METHODS AND ANALYSIS}

\section{Study design and setting}

We will conduct a two-phase mixed-method study at the CHU de Québec-Université Laval (Hôpital de l'EnfantJésus), a Canadian university-affiliated level 1 trauma centre with an annual total of 67000 visits. In this centre, the ED resuscitation/trauma team is activated by the triage nurse. All simulations will take place between March 2021 and October 2021 in the resuscitation/trauma area of the ED where real trauma patients are usually assessed on their arrival.

\section{Patient and public involvement}

Patients or the public were not involved in the design of this study, nor will they be involved in its conduct or in the reporting and dissemination of our research.

\section{Population}

ED health professionals from the CHU de QuébecUniversité Laval (Hôpital de l'Enfant-Jésus) will participate in the ISS training after informed verbal consent 
is obtained by research assistants. Teams of seven participants will be involved in each of the sessions (three nurses, two emergency physicians, a respiratory therapist and a resident). The emergency physicians are either Royal College emergency specialists (5-year training) or EM-trained family physicians (3-year training) from the Canadian College of Family Physicians. This team size and composition exactly match the trauma team that is activated when a real critical patient is admitted to the resuscitation/trauma area of our ED.

Phase 1 simulations will be announced and unannounced. Participants will be selected volunteers. ${ }^{27}$ During phase 2, participation will be random, as the sessions will be only unannounced.

\section{Scenario design}

Scenarios are inspired by real patients from a different ED in order to prevent participants from recognising real cases, which may lead to increased stress and unsolicited cognitive load for some ED professionals. ${ }^{28}{ }^{29}$ These scenarios will encompass common clinical presentations and will focus on two pathologies of interest for this ED: severe trauma (eg, traumatic brain injury, penetrating thoracic trauma, massive transfusion protocol activation) and cardiac arrest. The simulation team tested the scenarios beforehand during dedicated simulation training with a different population than the study participants. One of the key purposes for designing this study was to fulfil specific local teaching needs. Therefore, our tested training format will be useful to participants and could easily translate into improved patient care.

\section{Simulations}

For this study, we will use a Crash Kelly manikin from Laerdal (Laerdal Medical, Stavanger, Norway). Like other authors, we believe that the fidelity of the manikin itself is likely to be weakly correlated to the quality of learning, ${ }^{30} 31$ whereas the fidelity of the environment or of the scenario has a much greater probability of impacting the learning process. ${ }^{61532}$ We will use a thoracic prototype (created by one of the authors (CM)) to enhance realism and ensure flow immersion ${ }^{33}$ for the scenarios requiring thoracic invasive intervention (insertion of a chest tube, thoracotomy). The different health professionals included in our study have all been exposed to manikins, prototypes and simulation training of this type within the ED's sim lab. They were familiarised with this material through interprofessional training for the residents' simulation programme.

We will use real medications, with the exception of opioids and blood-derived products. If they are required for the simulation, we will reproduce blood products with saline bags coloured in red. For opioids, we will use saline with labels. We chose to use real medication to enhance realism and to better identify potential LST. Since training will take place in the trauma resuscitation area, the research team must limit the risks of mixing up real and fake medications.
In order to ensure patient safety and prevent disruptions to patient care in the ED during ISS, simulation experts have prepared a list of specific 'go/no go' criteria (see online supplemental material). These criteria are based on the existing literature on ISS in clinical settings such as the ED, ${ }^{3435}$ and we have adapted this list to some organisational specificities of our department. The 'no go' criteria include heavy clinical load, understaffing, low bed availability on wards and equipment needs (eg, unavailability of the fast flow fluid warmer). If a real trauma activation is expected or ongoing, the ISS will be cancelled, and the simulation team will leave the trauma room in its original state. This system will not only enhance realism but also ensure safety. ${ }^{35}$

The ISS training sessions will be short $-40 \mathrm{~min}$ in total $(20 \mathrm{~min} \text { of simulation and } 20 \mathrm{~min} \text { of debriefing })^{36}$-and will follow the classic briefing-simulation-debriefing format. ${ }^{37}$ Based on published reports ${ }^{36} 3839$ and our experience, keeping sessions and debriefings short greatly improves participant buy-in and reduces the impact on ED workflow. This approach was applied and successfully completed with a predefined objective of $30 \mathrm{~min} .{ }^{3638} 39$

Debriefing, an essential component of effective learning, will follow the plus delta model to optimise the educational impact for all participants. ${ }^{40}$ This short format offers the possibility of repetitive simulation training and debriefing, whereas most traditional forms of sim training are rarely available to complete interprofessional teams. ${ }^{11}$

During each ISS, no matter the format, LST identification will be performed by an external observer with a specific LST grid identification tool. ${ }^{18}$

\section{Procedure}

Phase 1

In the first phase of our study (figure 1), we will assess the feasibility and safety of implementing announced or unannounced ISS during working shifts in a busy ED. A mixed-method with a convergent design will be used. ${ }^{41}$ Both qualitative and quantitative data will be collected within a similar timeframe and will then be analysed separately and merged. Electronic study data will be kept in a password-protected file on the CHU de QuébecUniversité Laval's secure server. Paper data will be kept in a locked office within the CHU de Québec-Université Laval research centre. In order to protect participant identity, all data will be denominalised and participants will be identified using a research number. The key code linking

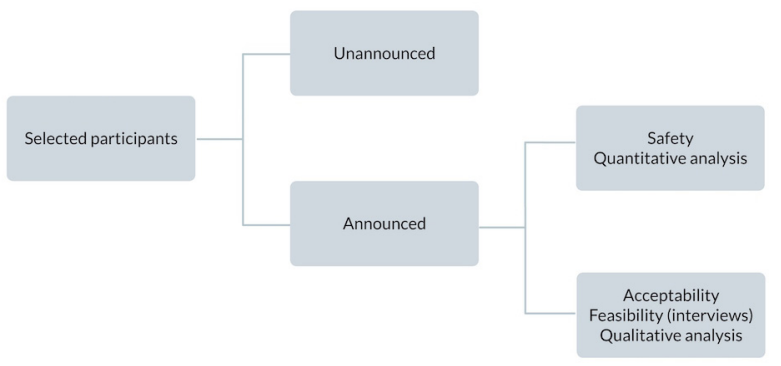

Figure 1 Phase 1. 
patient's name to the research number will be kept by the researcher in charge. All study data will be kept for 10 years and destroyed according to local modalities.

\section{Feasibility}

Feasibility will be assessed via semistructured individual interviews. Feasibility, according to Bowen $e t$ al, can be assessed through eight areas of focus: acceptability, demand, implementation, practicality, adaptation, integration, expansion and limited efficacy testing. ${ }^{42}$ Our study will assess the feasibility of each type of ISS with the following criteria: acceptability, implementation and practicality.

Acceptability and practicality, in particular, will be explored using semistructured individual interviews, which will take place after the exposition to ISS and cover topics based on our pre-established thematic framework (see online supplemental material). The themes of the semistructured interviews have been determined with the help of a qualitative research specialist and include previous professional experience and exposition to simulation, expectations, fear and thoughts regarding simulation training and assessment of a preference for an ISS format (announced or unannounced) (see online supplemental material). These interviews will take place at the end of phase 1.

Another aspect of feasibility, implementation (ie, the extent to which the simulation can be successfully conducted),${ }^{42}$ will be measured by compiling the number of cancelled sessions with a descriptive analysis of the circumstances and the reasons for cancellation.

The number of identified LSTs during announced and unannounced ISS will also be collected. We hypothesise that this number should be identical for both formats (announced or unannounced), and this is included in our feasibility analysis.

\section{Safety}

Safety is often an obstacle to ISS with working staff. ${ }^{43}$ Indeed, even though this factor is rarely assessed and included in the methodology of research projects exploring ISS, it is reasonable to fear that during an ISS training session-especially a long one-ED staff may neglect the other patients under their care. This may lead to patients leaving without being seen, or unnoticed adverse events leading to increased morbidity or mortality. Therefore, quantitative parameters measuring the impact of these training sessions on patient care will be collected: (1) the ED median wait time 6 hours before and 6 hours after ISS (stretcher and ambulatory care separately) by triage category and (2) the number of patients who left without being seen or against medical advice 6 hours after ISS. This information will be extracted from the institution's ED patient tracking software, and the data pertaining to the day of the unannounced ISS will be compared with that of the 3 days preceding the ISS, divided by working shifts $(8$ am to $4 \mathrm{pm}, 4 \mathrm{pm}$ to 12 am and 12 am to 8

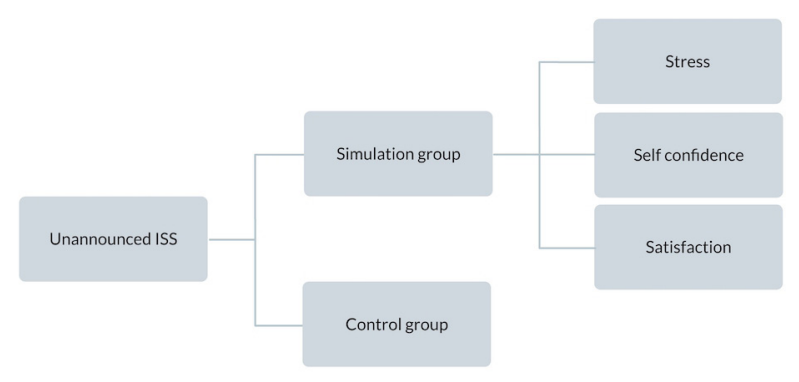

Figure 2 Phase 2.

am). Dedicated research staff will be present in the ED during the simulations and for up to 1 hour afterwards in order to record the occurrence of reported patientrelated adverse events (accident report) and the impact of the simulations on the working staff (understaffing and work overload). The research team will collect information regarding the impact of the training on the working staff using open-ended questions. Official accident reports will be collected and reviewed as needed, and unreported adverse events will be categorised by research staff.

\section{Phase 2}

Following phase 1, we will implement unannounced ISS-simulations that will take place during a work shift without advance notice to participants. This second phase (figure 2) will focus on assessing the impacts of unannounced ISS on health professionals as regards psychosocial risks such as stress, self-confidence and professional well-being using validated satisfaction and stress scales. ${ }^{44-46}$ The state anxiety questionnaire will be used along with the measurement of perceived stress, ${ }^{45}$ both validated for this type of methodology. The psychosocial risk assessment questionnaire and the assessment of self-confidence will reflect general professional wellbeing with validated assessment tools from the literature. ${ }^{47} 48$ In a recent study, simulation training provided a significant decrease in work stress among nurses in an intensive care unit. ${ }^{44}$ To demonstrate this benefit, staff members exposed to either unannounced or announced ISS training (ISS group) will be compared with those that were not exposed to ISS (control group) using the same questionnaires. The questionnaires will be filled out by every healthcare professional working in the participating ED at the end of phase 2 in order to compare the answers of the ISS group and the control group. Research assistants will collect the questionnaires with a tablet and ensure the completeness of our results.

\section{Outcomes}

Primary outcome:

Phase 1: proportion of successful ISS and qualitative exploration of feasibility among the two groups: announced and unannounced.

Phase 2: psychosocial risk levels among the two groups: ISS and no ISS (control). 
Secondary outcomes:

Phase 1:

- Quantitative patient safety parameters (wait times, adverse events, departures without being seen).

- Number of LSTs among the two groups: announced and unannounced.

Phase 2:

- Self-confidence levels among the two groups: ISS and no ISS.

- Stress levels among the two groups.

\section{Analyses}

Number of sessions

During phase 1 , a total of 16 sessions will be required (eight announced, eight unannounced) with a total of 112 participants. This number is in line with the previous literature in this field. ${ }^{25}$

We will conduct semidirected interviews until we reach data saturation, with a view to including sufficient variety in the different sociodemographic characteristics of participants.

During phase 2, a total of 10 unannounced ISS will be required to compare the group of participants exposed to ISS $(n=70)$ to the professionals not exposed to ISS $(n=70)$. Based on previous publication, ${ }^{44}$ this sample size will allow the detection of a $10 \%$ difference of the psychological demand score between groups (alpha: 0.05, power: 0.8).

\section{Thematic content analyses}

Audio-taped interviews will be transcribed, and thematic content analysis will be performed by two independent evaluators using a deductive approach guided by the semistructured interview's themes. The evaluators will follow Braun and Clarke's proposed six phases of thematic analyses. ${ }^{49}$ We will use NVivo V.12 Pro software. We have chosen to use the widely accepted and recognised criteria outlined by Lincoln and Guba to illustrate the quality of our study. ${ }^{50}$ To achieve credibility, we will employ peer debriefing to provide an external validation of the research process. Participants will be given chance to review the data collected by interviewers and the data's interpretations (member checking). This will offer the participants the opportunity to verify their statements and fill in any gaps from earlier interviews. We will provide thick descriptions to ensure transferability. To demonstrate dependability, we will ensure that the research process is logical, traceable and clearly documented. To achieve conformability, we will include markers such as the reasons for theoretical, methodological and analytical choices throughout the entire study, so that others can understand how and why decisions were made. A qualitative research expert and teacher from our university will be involved in each step of our research to provide guidance and ensure all of the above-mentioned quality control parameters.

\section{Quantitative analyses}

The quantitative analyses will include comparisons between the different participating groups. Continuous data will be expressed as an average (SD) when they are normally distributed and as a median (IQR Q1-Q3) otherwise. Continuous data will be analysed by paired t-tests, Wilcoxon signed rank tests and multiple linear regression, if applicable. The categorical variables will be expressed in numbers (percentages). Categorical variables will be compared using the $\chi^{2}$ test or the Fisher's exact test and logistic regression, if applicable. Bonferroni correction of multiple comparisons will be made. SAS statistical software will be used for all statistical analysis.

\section{Ethics and dissemination}

The CHU de Québec-Université Laval Research ethics board approved our study (number: 2020-5000). Potential participants in this study will receive an information form via email and in person before the announced ISS training. This information form will be distributed to every emergency professional and will include the research team's contact information should they have any question or should they refuse to participate in the study. Verbal consent will be obtained, and participants can withdraw at any time. The risks of participating in the study are no higher than when providing routine care to patients and/or during simulation training.

\section{Limitations}

Our study has some methodological limitations, most of which are inherent to simulation studies. ISS is new to the study site's ED. Therefore, to improve acceptance from the professionals, we have limited the number of announced and unannounced simulation to eight each. Therefore, we will conduct eight announced and eight unannounced ISS, excluding the cancelled sessions. As each session will involve seven participants, the total numbers of participants should be a minimum of 112 emergency professionals. After phase 1, we will adapt the number of ISS to the results obtained from our qualitative analysis. We accepted the selection bias created by the selection of volunteer participants during phase 1. As the aim is to validate our concept, the recruitment of motivated volunteers from the ED staff seemed to be an acceptable limit to the generalisation of our results. However, identifying and preparing selected participants is a widely accepted practice and is also recommended by change implementation experts.

For obvious reasons, randomising participants would not have been ethically acceptable. It was also impossible to blind participants to the outcomes of the study because the information form indicated they would have to fill out questionnaires and undergo semidirected interviews. However, the analysis and group comparisons will respect allocation concealment. The statistician will be blinded to the nature of the intervention, and the research staff conducting the safety analysis and the qualitative analysis will be blinded to the 'announced or unannounced' nature of the intervention. 
It was difficult to find the optimal compromise between holding short, pragmatic and acceptable ISS training sessions (to limit the risks of negatively impacting ED operations) and maintaining educational objectives to ensure effective learning. With these considerations in mind, we will choose 'quieter' moments of the day to hold the simulations and will, therefore, be unable to reproduce the inherent chaos of the ED with perfect realism. Still, we feel this is an ethical imperative for a research team wishing to conduct ISS in a busy ED.

\section{Dissemination strategy}

This is the first scientific work to assess both feasibility and participant-centred outcomes. It is, therefore, an original, unexplored training situation, which may be associated with a practical clinical impact. ISS is a practical and safe teaching method that suits the specific constraints and needs pertaining to EM. In addition, one of the main limits to the wide implementation of simulation is its high cost. If ISS proves feasible in the ED, it could reduce the costs inherent to the structure (simulation centre) and associated human resources while increasing the safety of the process. Assessing the feasibility of a new intervention such as ISS and taking into account the opinions of the professionals involved will facilitate future implementation and uptake by targeted users. The impact of these training sessions on patient care could be measured through simple epidemiologic data collection. Improved care for severe trauma patients would also translate into lower public health costs. In addition, few studies exist on the importance of realism in simulation, but the importance of training in conditions close to real practice has already been shown. ${ }^{32}$ For all these reasons, we are working in close partnership with important knowledge users from our institution towards a single, shared goal to improve patient safety. Publications in peer-reviewed journals and international conferences presentations are also planned.

\section{Twitter Marcel Emond @marcel_mond}

Acknowledgements The authors would like to thank Steve Paquet, Jolene Provost, Joannie Blais, and Hugh Scott and the Hôpital de l'Enfant-Jesus' emergency physician for their support and implication in this study.

Contributors JT led the development of the protocol and drafted the manuscript. VB, ER-D and MÉ drafted the manuscript with the first author. CM, EB, JM, GM, GC, $\mathrm{CG}$ and $\mathrm{GB}$ critically revised and approved the final version of the manuscript.

Funding This work was supported by the Fondation du CHU de Québec-Université Laval, grant number 3967. JT received a Fellowship award from the Société française de médecine d'urgence. The sponsors were in no way involved in the design of this study protocol.

Competing interests None declared.

Patient consent for publication Not required.

Provenance and peer review Not commissioned; externally peer reviewed.

Supplemental material This content has been supplied by the author(s). It has not been vetted by BMJ Publishing Group Limited (BMJ) and may not have been peer-reviewed. Any opinions or recommendations discussed are solely those of the author(s) and are not endorsed by BMJ. BMJ disclaims all liability and responsibility arising from any reliance placed on the content. Where the content includes any translated material, BMJ does not warrant the accuracy and reliability of the translations (including but not limited to local regulations, clinical guidelines, terminology, drug names and drug dosages), and is not responsible for any error and/or omissions arising from translation and adaptation or otherwise.

Open access This is an open access article distributed in accordance with the Creative Commons Attribution Non Commercial (CC BY-NC 4.0) license, which permits others to distribute, remix, adapt, build upon this work non-commercially, and license their derivative works on different terms, provided the original work is properly cited, appropriate credit is given, any changes made indicated, and the use is non-commercial. See: http://creativecommons.org/licenses/by-nc/4.0/.

ORCID iD

Marcel Emond http://orcid.org/0000-0001-7158-8110

\section{REFERENCES}

1 Cook DA. How much evidence does it take? A cumulative metaanalysis of outcomes of simulation-based education. Med Educ 2014;48:750-60.

2 Cook DA, Brydges R, Zendejas B, et al. Technology-enhanced simulation to assess health professionals: a systematic review of validity evidence, research methods, and reporting quality. Acad Med 2013;88:872-83.

3 Cook DA, Hatala R, Brydges R, et al. Technology-enhanced simulation for health professions education: a systematic review and meta-analysis. JAMA 2011;306:978-88.

4 Aggarwal R, Mytton OT, Derbrew M, et al. Training and simulation for patient safety. Qual Saf Health Care 2010;19:i34-43.

5 Rehmann AJ, Mitman RD, Reynolds MC. A Handbook of flight simulation fidelity requirements for human factors research. Crew system ergonomics information analysis center Wright-Patterson AFB $\mathrm{OH}, 1995$.

6 Krogh KB, Høyer CB, Ostergaard D, et al. Time matters-realism in resuscitation training. Resuscitation 2014;85:1093-8.

7 DeMaria S, Silverman ER, Lapidus KAB, et al. The impact of simulated patient death on medical students' stress response and learning of ACLS. Med Teach 2016;38:730-7.

8 Philippon AL, Bokobza J, Bloom B, et al. Effect of simulated patient death on emergency worker's anxiety: a cluster randomized trial. Ann Intensive Care 2016;6:60.

9 Gaba DM. The future vision of simulation in health care. Qual Saf Health Care 2004;13:i2-10.

10 Marshall SD, Flanagan B. Simulation-based education for building clinical teams. J Emerg Trauma Shock 2010;3:360-8.

11 Calhoun AW, Boone MC, Peterson EB, et al. Integrated in-situ simulation using redirected faculty educational time to minimize costs: a feasibility study. Simul Healthc 2011;6:337-44.

12 McGaugh JL. Memory-a century of consolidation. Science 2000;287:248-51.

13 Bhanji F, Donoghue AJ, Wolff MS, et al. Part 14: education: 2015 American heart association guidelines update for cardiopulmonary resuscitation and emergency cardiovascular care. Circulation 2015;132:S561-73.

14 Anderson R, Sebaldt A, Lin Y, et al. Optimal training frequency for acquisition and retention of high-quality CPR skills: a randomized trial. Resuscitation 2019;135:153-61.

15 Durning SJ, Artino AR. Situativity theory: a perspective on how participants and the environment can interact: AMEE guide No. 52. Med Teach 2011;33:188-99.

16 Sørensen JL, van der Vleuten C, Rosthøj S, et al. Simulation-Based multiprofessional obstetric anaesthesia training conducted in situ versus off-site leads to similar individual and team outcomes: a randomised educational trial. BMJ Open 2015;5:e008344.

17 Sørensen JL, Navne LE, Martin HM, et al. Clarifying the learning experiences of healthcare professionals with in situ and off-site simulation-based medical education: a qualitative study. BMJ Open 2015;5:e008345.

18 Patterson MD, Geis GL, Falcone RA, et al. In situ simulation: detection of safety threats and teamwork training in a high risk emergency department. BMJ Qual Saf 2013;22:468-77.

19 Alfredsdottir H, Bjornsdottir K. Nursing and patient safety in the operating room. J Adv Nurs 2008;61:29-37.

20 Ben-Ari M, Chayen G, Steiner IP, et al. The effect of in situ simulation training on the performance of tasks related to patient safety during sedation. J Anesth 2018;32:300-4.

21 Lighthall GK, Poon T, Harrison TK. Using in situ simulation to improve in-hospital cardiopulmonary resuscitation. Jt Comm J Qual Patient Saf 2010;36:209-16.

22 Knobel A, Overheu D, Gruessing M, et al. Regular, in-situ, teambased training in trauma resuscitation with video Debriefing 
enhances confidence and clinical efficiency. BMC Med Educ 2018;18:127.

23 Wang CJ, Lin SY, Tsai SH, et al. Implications of long-term low-fidelity in situ simulation in acute care and association with a reduction in unexpected cardiac arrests: a retrospective research study. PLoS One 2019;14:e0213789.

24 Westbrook JI, Raban MZ, Walter SR, et al. Task errors by emergency physicians are associated with interruptions, multitasking, fatigue and working memory capacity: a prospective, direct observation study. BMJ Qual Saf 2018;27:655-63.

25 Freund D, Andersen PO, Svane C, et al. Unannounced vs announced in situ simulation of emergency teams: feasibility and staff perception of stress and learning. Acta Anaesthesiol Scand 2019;63:684-92.

26 Paltved C, Bjerregaard AT, Krogh K, et al. Designing in situ simulation in the emergency department: evaluating safety attitudes amongst physicians and nurses. Adv Simul 2017;2:4.

27 Powell BJ, Waltz TJ, Chinman MJ, et al. A refined compilation of implementation strategies: results from the expert recommendations for implementing change (ERIC) project. Implement Sci 2015;10:21.

28 Chiniara G, Cole G, Brisbin K, et al. Simulation in healthcare: a taxonomy and a conceptual framework for instructional design and media selection. Med Teach 2013;35:e1380-95.

29 Brydges R, Carnahan H, Rose D, et al. Coordinating progressive levels of simulation fidelity to maximize educational benefit. Acad Med 2010;85:806-12.

30 Cheng A, Lockey A, Bhanji F, et al. The use of high-fidelity manikins for advanced life support training--A systematic review and metaanalysis. Resuscitation 2015;93:142-9.

31 Hamstra SJ, Brydges R, Hatala R, et al. Reconsidering fidelity in simulation-based training. Acad Med 2014;89:387-92.

32 O'Leary F, Pegiazoglou I, McGarvey K, et al. Realism in paediatric emergency simulations: a prospective comparison of in situ, low fidelity and centre-based, high fidelity scenarios. Emerg Med Australas 2018;30:81-8.

33 Czsentmihalyi M. Flow: the psychology of optimal experience. Harper Perennial, 1990.

34 Bajaj K, Minors A, Walker K, et al. "No-Go considerations" for In Situ simulation safety. Simul Healthc 2018;13:221-4.

35 Raemer D, Hannenberg A, Mullen A. Simulation safety first: an imperative. Simul Healthc 2018;13:373-5.

36 Wheeler DS, Geis G, Mack EH, et al. High-reliability emergency response teams in the hospital: improving quality and safety using in situ simulation training. BMJ Qual Saf 2013;22:507-14.
37 Granry J, Moll M. Rapport de mission de la Haute Autorité de Santé. État de l'art (national et international) en matière de pratiques de simulation dans le domaine de la santé St. Denis la Plaine [online]. Haute Autorité de santé, 2012. www.has-sante.fr

38 Surcouf JW, Chauvin SW, Ferry J, et al. Enhancing residents' neonatal resuscitation competency through unannounced simulationbased training. Med Educ Online 2013;18:18726-7.

39 Petrosoniak A, Auerbach M, Wong AH, et al. In situ simulation in emergency medicine: moving beyond the simulation lab. Emerg Med Australas 2017;29:83-8.

40 Bajaj K, Meguerdichian M, Thoma B, et al. The PEARLS healthcare debriefing tool. Acad Med 2018;93:336.

41 Fetters MD, Curry LA, Creswell JW. Achieving integration in mixed methods designs-principles and practices. Health Serv Res 2013;48:2134-56.

42 Bowen DJ, Kreuter M, Spring B, et al. How we design feasibility studies. Am J Prev Med 2009;36:452-7.

43 Auerbach M, Roney L, Aysseh A, et al. In situ pediatric trauma simulation: assessing the impact and feasibility of an interdisciplinary pediatric in situ trauma care quality improvement simulation program. Pediatr Emerg Care 2014;30:884-91.

44 El Khamali R, Mouaci A, Valera S, et al. Effects of a multimodal program including simulation on job strain among nurses working in intensive care units: a randomized clinical trial. JAMA 2018;320:1988-97.

45 Bellinghausen L, Collange J, Botella M, et al. Validation factorielle de l'échelle française de stress perçu en milieu professionnel. Santé Publique 2009;21:365-73.

46 Spielberger C, Gorsuch R. Inventaire d'anxiété, état-trait : forme $Y$ (STAI-Y) [adapt. française de M. Bruchon-Schweitzer et de I. Paulhan] Paris : Ed. du Centre de psychologie appliquée. Catalogue général Bibliothèque nationale de France, 1993. https://catalogue.bnf.fr/ark:/ 12148/cb355889031

47 Vallieres EF, Vallerand RJ. Traduction et validation CanadienneFrançaise de L'échelle de L'estime de Soi de Rosenberg. Int J Psychol 1990;25:305-16.

48 É D, Bocéréan C, Teherani M. Le COPSOQ: un nouveau questionnaire Français d'évaluation des risques psychosociaux. Santé publique 2012;24:189-207.

49 Braun V, Clarke V. Using thematic analysis in psychology. Qual Res Psychol 2006;3:77-101.

50 Guba EG, Lincoln YS. Fourth generation evaluation. Sage, 1989.

51 Lincoln YS, Guba EG, Pilotta JJ. Naturalistic inquiry. Int J Intercultural Relation 1985;9:438-9. 